\title{
The Effect of Phi on the Perception of Attractiveness
}

\author{
Adrien Wilson-Thompson ${ }^{1}$ \\ ${ }^{1}$ Eleanor Roosevelt High School
}

\begin{abstract}
$\underline{\text { ABSTRACT }}$
The ratio Phi, equal to approximately 1.618, is an observable phenomenon that Greek mathematician Euclid defined this relationship as a line in two parts, in which the ratio of the longer part to the shorter part is equal to the ratio of the total length over the longer part. The ancient Greeks thought of Phi as a mathematical representation of physical beauty. Although many studies have shown a correlation between Phi and perceived attractiveness, other studies have displayed no/very weak, correlation. The question, "Does the expression of Phi play a significant role in the attractiveness of a face?" was investigated. It was hypothesized that if the features of a face closely fit the proportions of Phi, then survey respondents will give that face a higher attractiveness rating than a face whose features do not. Landmark localization data was calculated for images of 14 different faces. Individual and average facial ratios were calculated for each photograph, and a survey was conducted in which 100 participants were asked to rate the attractiveness of each face on a scale of 1-10. The average attractiveness rating of each face was then compared to the variance/distance of its average facial ratio away from the numerical value of Phi. Based on the analysis of the data, faces with features whose proportions closely fit the ratio of Phi are perceived as more attractive than faces with features that do not. Faces with average facial ratios closer to Phi received higher average attractiveness ratings.
\end{abstract}




\section{Chapter One}

\section{The Problem and Its Setting}

In the context of facial aesthetics, it is believed by some that a mathematical proportion, called Phi $(\Phi)$, can be used to measure the attractiveness of a face. The ancient Greeks believed that Phi, denoted by the specific ratio 1:1.618, embodied the representation of physical beauty (Pallett, et al., 2010). Some studies indicate a distinct correlation between Phi and perceived attractiveness. Conversely, other studies dispute these claims, and reveal no, or very weak, correlation. If correlation is found through further study, the results can be applied to define a universal standard of attractiveness, and could potentially be used as a basis for ideal facial aesthetics in cosmetic and facial reconstructive procedures.

\section{Background of the Study}

In the late fourth century, Greek mathematician Euclid defined the relationship of Phi as being a line in two parts (a, $b)$, in which the ratio of the two parts $(\mathrm{a} / \mathrm{b})$ is equal to the ratio of the total length over the longer part (a+b/a) (Kaya, et al., 2018). Phi, also referred to as the Golden Ratio, is commonly observed throughout nature and history. The ratio can be identified in various historical structures and works of art, such as the Parthenon, the Great Pyramid, and the Mona Lisa. It is also found in the natural world, such as within the cochlea of the human ear, the arrangement of fern leaves, and in the spirals of nautilus shells.

\section{Statement of Problem}

The aim of this study was to examine whether a relationship between Phi and perceived facial beauty exists. If correlation can be identified, the ratio of Phi could potentially be applied to define a universal standard of attractiveness, and used as a guide for enhancing facial reconstructive procedures and other cosmetic surgery applications. Specifically, the research question "Does the expression of Phi play a significant role in the attractiveness of a face?" was studied.

\section{Hypothesis}

If the features of a face closely fit the proportions of Phi, then survey respondents will give that face a higher attractiveness rating than a face whose features do not. The null hypothesis stated that there is no correlation between Phi and perceived facial attractiveness.

\section{Variables}

Independent Variable

The variance of a face's proportions from the numerical value of Phi

Dependent Variable

Attractiveness rating 


\section{Control Group}

Not applicable

\section{Regulated Conditions}

Images displayed

Order of images

Time allotted to view images

\section{Assumptions and Limitations}

\section{Assumptions}

Survey respondents' ratings are subjective in nature and largely reflective of their individual preferences

Survey respondents report honest answers in terms of personal preference, not what they believe is the correct answer relative to Phi values

\section{Limitations}

All survey respondents are within the same age range, and not varied

Test images are all of the same race, leading to limited test pool

\section{Statistical Analysis}

A Pearson's Correlation Coefficient Statistical Test was utilized for the study's quantitative data analysis at an $\alpha=.01$ significance level. The null hypothesis states that there is no correlation between Phi and facial attractiveness, resulting in an $r^{2}$ value of zero between the examined relationships.

\section{Summary}

This study was intended to identify whether a correlation exists between Phi and perceived facial attractiveness. The question "does the expression of Phi play a significant role in the attractiveness of a face?" was studied by examining the effect that the variance of a face's proportions from the numerical value of Phi has on its average attractiveness rating. Through the use of a Pearson's Correlation Coefficient Statistical Test at an $\alpha=.01$ significance level to analyze the data, the null hypothesis, which states that there is no correlation between the examined relationships, could either be rejected or fail to be rejected.

\section{Definitions of Terms and Abbreviations}

1. Aesthetic - of or relating to beauty or pleasing appearance

2. Beauty Standards - socially constructed ideals in regard to beauty and attractiveness

3. Cheilion $(\mathrm{Cn})$ - point in corner of the mouth

4. Facial Morphology - shape and structure of a face

5. Gnathion $(\mathrm{Gn})$ - the lowest point in the middle of the soft tissue of the menton

6. Lateral canthus (Lc) - point in lateral canthus of eyes

7. Lateral nasal (Ln) - outermost point of the nostril

8. Menton $(\mathrm{Mn})$ - most interior point of the chin 
9. Phi - also called Golden Ratio/Divine Proportion; proportion that relates to beauty (the longer part divided by the smaller part equals the whole length of the given line segment divided by the longer part)

10. Subnasale (Sn) - the intersection point of the upper lip and nasal septum

11. Temporal soft tissue (TST) - arches of the eyebrows

12. Trichion $(\mathrm{Tr})$ - junction point of the upper part of the forehead

13. Zigoma (Zy) - lateral point of the zygomatic arc 


\section{Chapter Two}

\section{The Review of the Related Literature}

The ratio of Phi $(\Phi)$ has been commonly observed throughout nature and history. In the late fourth century, Greek mathematician Euclid defined this relationship as being a line in two parts (a,b), in which the ratio of the two parts (a/b) is equal to the ratio of the total length over the longer part (a+b/a) (Kaya, et al., 2018). The ancient Greeks believed that Phi, also referred to as the Golden Ratio, could be used to measure physical beauty and attractiveness. Many studies have shown a distinct correlation between Phi and perceived beauty. In contrast, other studies have disputed these claims, and have shown no, or very weak, correlation.

This experiment aimed to examine the possible relationship between Phi and perceived facial attractiveness by comparing ratings of various test faces to their calculated Phi ratios. The relationship between Phi and perceived facial beauty has long been a topic of debate, with countless studies either proving or disproving the relationship. In order to assess the implications of perceived beauty, the definition of beauty and beauty standards and their societal impacts must first be investigated. To analyze the significance of the role of Phi in perceived attractiveness, the definition and common measurements of Phi, along with the supporting and contradictory studies, must be examined. If correlation is identified and a relationship can be determined, Phi could be applied to define a universal standard of attractiveness. This standard could then be utilized as a guide for surgical facial reconstructive procedures, as well as for other cosmetic applications.

\section{Definition of Beauty}

Beauty is a personalized and subjective construct. "Beauty is in the eye of the beholder" is an age-old axiom that attests to the subjective nature of it (Schmid, et al., 2008). According to Pamela M. Pallett, a senior researcher at the University of California San Diego, "the quest to define facial beauty either by the size or shape of isolated facial features... or by the spatial relations between facial features dates back to antiquity" (Pallett, et al., 2010). Throughout history, varying philosophies existed on the true meaning of beauty. For example, Greek philosopher Aristotle believed that the goal of all virtue is beauty, while Prussian philosopher Kant considered beauty a symbol of moral goodness (Cui, et al., 2019). Modern research into the perception of beauty has identified many different factors that contribute to attractiveness. Some studies suggest that beauty cannot be defined by one single principle, whereas other studies claim that attractiveness is centered on "averageness, symmetry, and sexual dimorphism" (Schmid, et al., 2008). Additional studies suggest that perceived attractiveness of oneself influences one's perception of the attractiveness of others, whereas other studies claim that people tend to prefer faces that resemble their own (Schmid, et al., 2008).

\section{Beauty Standards}

Standards of beauty are not static, but instead have evolved throughout history. During the Renaissance period, painters and sculptors used neoclassical canons as guides to artistically represent "perfect" faces. Neoclassical cannons are defined ratios that Renaissance artists believed to be optimal facial proportions (Schmid, et al., 2008). Examples of neoclassical cannons include the length of the nose being equal to the length of the ear, the distance between the eyes being equal to the width of the nose, and the width of the face being four times the width of the nose (Schmid, et al., 2008). Symmetry has also been considered to be an important factor in perceived attractiveness. In reference to facial beauty, only symmetry about the vertical axis is taken into consideration (Schmid, et al., 2008). 
Recent research has suggested that set ideals exist that can be utilized to determine the attractiveness of a face. Researchers, Dr. José Francisco Ballester Ferrandis, Prof. Francisco Martinez Soriano, Dr. Maria Isabel Ribera Vega, and Dr. Juan José Font Ferrandis, performed a study at the University of Madrid that was instrumental in defining universal beauty standards. Participants in this study were asked to rate photographed faces according to their degree of attractiveness on a scale of "very ugly" to "very good looking" (Ballester, et al., 2018). After the initial ratings were recorded, the researchers then asked a series of survey questions to determine how the ratings were chosen. $100 \%$ of the participants who rated a face as being "ugly" or "very ugly" reported that they thought the eyebrows were too close together (Ballester, et al., 2018). In addition, $57 \%$ of the participants that considered a face to be "very ugly" thought that the eyes were too small and/or sunken, whereas $100 \%$ of the participants who thought a face was "very good looking" reported that the eyes were "normal" sized (Ballester, et al., 2018). Furthermore, $80 \%$ of the people who reported a face as being "ugly" or "very ugly" reported that the nose was "very wide," whereas $75 \%$ of the people who said a face was "very good looking" reported the nose as being "very narrow" (Ballester, et al., 2018). The study concluded that a balanced face for males is comprised of a slightly narrow nose, small eyes which are not sunken, protruded lips, and a slightly protruding chin; while a balanced face for females is comprised of a narrow nose, big eyes which are not sunken, and very protruded lips (Ballester, et al., 2018).

\section{Societal Impacts of Attractiveness}

Physical appearance and attractiveness has a clear and observed impact within society. How attractive an individual is "plays a major role in determining the outcomes of one's life-course trajectory including vocational success, social relationships, mental health, and overall well-being" (Foos, et al., 2011). The face is arguably one of the most important factors affecting the physical appearance of a person (Nguyen, et al., 2016). It is an established psychological principle that humans prefer attractive faces to unattractive ones, and this preference is observed from early infancy, and is consistent across age, gender, and ethnicity (Pallett, et al., 2010). Human preference for attractiveness stems from "evolutionary functional assessments and evaluations of potential mates" (Eisenthal, et al., 2006). With Darwinian principles in mind, attractive faces are a biological signal of overall value as a mate (Eisenthal, et al., 2006). Characteristics such as good skin quality and bone structure are associated with good health, and therefore unconsciously contribute to the perception of attractiveness (Eisenthal, et al., 2006). Physical appearance has also been shown to have an effect on the collective judgment of moral character, known as the "beauty-is-good" stereotype (Cui, et al., 2019). People who are perceived as being significantly attractive are typically assumed to be more kind, honest, and moral, when compared to others (Cui, et al., 2019). Research has shown that individuals who are considered significantly attractive have an overall advantage in life, and the search to find a formal definition of beauty and attractiveness is an ongoing and contentious topic of research and debate.

\section{Definition of Phi}

The Ancient Greeks believed that beauty was represented by a specific ratio of parts to a whole, known as Phi $(\Phi)$, which equals 1:1.618 (Pallett, et al., 2010). Greek Mathematician Euclid stated, "a straight line is said to have been cut in extreme and mean ratio when, as the whole line is to the greater segment, so is the greater to the lesser," and was the first to define this relationship (Özdemir, et al., 2019). This relationship is expressed mathematically as: "(a $+\mathrm{b}) / \mathrm{a}=\mathrm{a} / \mathrm{b}=\Phi$ " (Ilić et al. 2018). In the late 20th century, researcher Mark Barr coined the term "Phi," and its symbol $\Phi$, in commemoration of the Greek scientist and mathematician Phidias to refer to this relationship (Özdemir, et al., 2019). This ratio can be observed in numerous architectural works and natural occurrences, from the Parthenon and the Great Pyramids, to nautilus shells and the cochlea of human inner ear (Ilić, et al., 2018). Additionally, shapes that exhibit the ratio of Phi have been shown to be more aesthetically pleasing to the eye than others (Fletcher, 2007). Faces with features that conform to the ratio of Phi are believed to be aesthetically pleasing regardless of sex or race 
(Veerala, et al., 2016). In the context of perceived beauty and attractiveness, the ratio of Phi is also referred to as the Golden Ratio.

\section{Measurements of Phi}

When measuring the existence of Phi in facial features, many different relationships can be observed. Some studies use a holistic approach, using points from various areas of the face, to test for the occurrence of Phi. Researchers at Danang University of Technical Medicine and Pharmacy performed an experiment to study facial aesthetics through analyzing the relationships between the Trichion, the Lateral canthus, the Zigoma, the Subnasale, the Cheilion, and the Gnathion (Nguyen, et al., 2016). These points on the face are key landmarks that are analyzed in many investigations related to Phi. Researchers at the University of Tuebingen Medical School also used measurements between the Trichion, Subnasale, Gnathion, and Zigoma to investigate the relationship between facial proportions and Phi (Özdemir, et al., 2019). Other studies, however, chose to focus on vertical and horizontal measurements of facial features in assessing Phi. Researchers at the University of California, San Diego, focused on the vertical distance between the eyes and the mouth, and the horizontal distance between the pupils, to investigate the occurrence of Phi in relation to the distance and width of a face (Pallett, et al., 2010).

\section{Supporting Studies}

Phi is thought to evoke an aesthetically pleasing effect (Alam, et al., 2015). In the previously mentioned study performed by Dr. José Francisco Ballester Ferrandis and his team at the University of Madrid, the ratio of Phi was observed in every face that was considered "very attractive" (Ballester, et al., 2018). The data collected by the team revealed, "with 99\% confidence, the golden ratio was within virtually every interval" (Ballester, et al., 2018). The team concluded that there is in fact "a general correlation between specific facial proportions and the golden ratio" (Ballester, et al., 2018).

Researchers Valentin Buşuat and Elena Andrei of the University of Craiova performed an additional study that upheld the relationship between Phi and perceived attractiveness. The study was a qualitative research-based investigation into the significance of facial aesthetics in modern society, in which they analyzed the relationship between the appearance of Phi in facial reconstructive surgeries and levels of satisfaction reported by patients. Their study concluded, "society's great importance on the aesthetic-facial aspect... has led to an increase in demand for cosmetic surgery," and, "that surgery found to be more in line with the golden ratio increases self-esteem" (Bussuat \& Andrei, 2018).

Dr. Mounir Bashour, $\mathrm{PhD}$ from the University of Toronto, presented the argument that people from different cultures and backgrounds rate faces for attractiveness on similar qualities, and proposed the theoretical existence of a universal model for objectively rating facial attractiveness (Bashour, M., 2016). In his study, Dr. Bashour took pictures of 72 subjects and individually marked them to display 224 predefined feature points in accordance with markers of the Phi Mask developed by Dr. Stephen Marquardt. The Phi Mask is a representation of the arrangement of facial features that are in perfect harmony with the Golden Ratio. The pictures were then rated by volunteer participants on a scale of 1-10, with 10 being extremely attractive. Dr. Bashour used the Phi mask model as a template for denoting attractiveness, and created a quantitative system by measuring the numerical divergence of the facial landmarks from their equivalent mask nodal points (Bashour, M., 2016). The average of the ratings given to the faces that were closer to Phi proportions was higher than those of the faces that deviated from Phi proportions. He concluded that there is a "direct correlation of mask deviation score to attractiveness quotient assigning equal weightings to deviations from each of the anthropometric landmarks shows that the Phi mask does indeed work to give an objective measure of facial attractiveness" (Bashour, M., 2016). 


\section{Contradicting Studies}

There is also extensive research into the relationship between Phi and perceived attractiveness that shows no correlation between the two. A study performed by researchers at the Department of Orthodontics at Istanbul Medipol University aimed to determine whether attractive people have normal or ideal facial relationships that exhibit a correlation to Phi (Akan, et al., 2017). The researchers took images of 335 test subjects, which were then randomly placed into a presentation to display to the "judges" of the experiment (Akan, et al., 2017). The judges scored the images from 1 (least attractive) to 10 (most attractive) according to their own preferences. The 30 images with the highest scores and the 30 images with the lowest scores were selected for further evaluation. To investigate the Phi ratios of the attractive and non-attractive subjects, 13 landmarks and 12 linear measurements were used on the photographs (Akan, et al., 2017). The researchers found that there was no statistical difference between the attractiveness scores and their relation to the Golden Ratio, and they concluded that Phi is not a universal scale of attractiveness (Akan, et al., 2017).

Researchers at the Orthodontic Unit in the School of Dental Science at the University of Malaysia conducted a study that aimed to "provide a facial anthropometric and aesthetic analysis" of the association of facial proportion to Phi among the Malaysian population (Alam, et al., 2015). The study involved 268 total participants of ages 18-25, and observed four specific facial measurements: "total facial height (Tr-Me), upper facial height (Tr-Gb), middle facial height (Gb-Sn) and lower facial height (Sn-Me) as well as width of face (Zy-Zy)" (Alam, et al., 2015). The data revealed no statistically significant relationship between Phi and facial proportions. The researchers concluded, "generally, the majority of investigations reported a weak correlation between golden proportion and attractiveness" (Alam, et al., 2015).

Finally, a study by researchers at the Danang University of Technical Medicine and Pharmacy was performed to evaluate proportions of frontal facial soft-tissues in relation to Phi and perceived beauty, specifically within the female Vietnamese population, ages 19-25. Photographs of the participants were taken, and the Trichion, Temporal soft tissue right and left, Lateral Canthus right and left, Cheilion right and left, and Menton measurements were collected (Nguyen, et al., 2016). The study involved a survey in which they asked participants to rate the pictures, and then compared the survey results to the facial morphology measurements. They found that the facial proportions of perceived attractive Vietnamese females displayed no statistical relationship to Phi (Nguyen, et al., 2016).

\section{Summary}

Standards of beauty have meaningful societal impacts. Individuals who exhibit significant facial attractiveness are reported to experience an overall better quality of life than individuals who do not. The ratio of Phi was historically hypothesized to represent an indication of beauty. Numerous studies exist which indicate a correlation between Phi and perceived attractiveness. Conversely, other studies exist that contradict these findings. There is no formally accepted definition or measurement of beauty, but if correlation is identified, and a relationship can be determined, the measurements of the ratio of Phi could be used to establish a universal standard of attractiveness. 


\section{Chapter Three}

\section{Method}

Phi $(\Phi)$, equal to approximately 1.618 , is theorized as providing a basis for measuring and analyzing attractiveness and beauty. If correlation between Phi and perceived facial attractiveness can be identified, Phi could potentially be used as a basis for achieving ideal facial aesthetics in cosmetic and facial reconstructive procedures.

Since studies have shown both the existence and nonexistence of a distinct correlation between Phi and attractiveness, this experiment aimed to achieve a better understanding of the possible relationship. Specifically, the question "Does the expression of Phi play a significant role in the attractiveness of a face?" was examined, and it was hypothesized that survey respondents would rate the attractiveness of a face higher the more closely that face's proportions aligned with Phi.

\section{Materials}

Aberdeen 2D Face Set from the Psychological Image Collection at Stirling (PICS) Database

14 Images; Caucasian (7 male/ 7 female); Ages 18-40

Pixel Measurement Software

RapidTables.com

Timer

\section{Procedures}

For the purposes of this experiment, only images that showed a full-frontal view of a face, with little to no facial expression, were selected. All images chosen were of Caucasian descent, and did not include glasses, jewelry, or other inconsistencies in order to reduce the variability among the images. Participants of the survey were between the ages of 14-18, and the study was performed at Eleanor Roosevelt High School.

\section{Landmark Localization Procedure}

I. Set of faces selected from Aberdeen 2D Face Set from the Psychological Image Collection at Stirling (PICS) Database
a. Images 1-14 (Appendix)

II. Mark facial landmarks for each image

a. Chart 3.1 (Appendix)

III. Calculate individual ratio values for each image based on facial landmarks using RapidTables.com pixel measurement software

a. Chart 3.2 (Appendix)

IV. Calculate the average of the ratio values (average Phi value) for each image

\section{Survey Procedure}

I. Ensure participant is in stimulus-free testing area

II. Show first image 
III. Give participant 10 seconds to rate the attractiveness of the image on a scale of 1-10:
a. 10 - Extremely Attractive
b. 9 - Very Attractive
c. 8 - Attractive
d. 7 - Mildly Attractive
e. 6 - Neutral Plus
f. 5 - Neutral Minus
g. 4 - Mildly Unattractive
h. 3 - Unattractive
i. 2 - Very Unattractive
j. 1 - Extremely Unattractive

IV. Record participant's response

V. Display next image

VI. Repeat steps III-V for each image

VII. Repeat steps I-VI for each participant

\section{Data Collection and Analysis}

The independent variable in this experiment was the average Phi value of each image, and the dependent variable was the attractiveness rating given by each survey participant. The regulated conditions in this experiment were the test pictures, the facial landmarks, the measurement software, the order in which the images were shown, and the allotted time to view each image. Data was collected in the form of a survey in which participants were asked to rate each image on a 10-point scale (10 being the most attractive, 1 being the least attractive). The Pearson Correlation Coefficient statistical test was used to analyze the data.

\section{Summary}

This study was designed to observe the effect of Phi on perceived attractiveness. Images from the PICS database were selected and marked to display key facial landmarks (Chart 3.1), which were used to calculate individual ratio values (Chart 3.2) for each image. The average of the individual ratio values was then calculated for each image, and was referred to as the average Phi value. In the survey, participants were asked to rate each face on a scale of 1-10. The ratings were then compared to their average Phi values to establish whether a correlation exists between average Phi values close to 1.618 and attractiveness ratings close to 10 . 


\section{Chapter Four}

\section{The Findings}

The aim of this experiment was to investigate and test for a relationship between Phi and perceived facial beauty. The question "does the expression of Phi play a significant role in the attractiveness of a face?" was studied. It was hypothesized that if the features of a face closely fit the proportions of Phi, then participants will give that face a higher attractiveness rating than a face with features that do not. It was anticipated that the survey respondents would favor faces that more closely align with Phi.

\section{Data}

Landmark localization data for each of the 14 photographs were collected using RapidTables pixel measurement software. The landmark localization ratio values were calculated based on pixels. Individual ratios were calculated for each face, and then average values were determined (see Appendix: Table 1-14). In the survey, 100 participants were asked to rate the attractiveness of each face on a scale of 1-10. The average attractiveness rating was found by calculating the mean of the survey responses for each face (see Appendix: Table 17). The data from the landmark localization averages and the survey results were expressed as distinct relationships in two tables: "Variance from Phi vs. Average Attractiveness Rating" and "Distance from Phi vs. Average Attractiveness Rating." The variance values were calculated by subtracting 1.618 (Phi) from the Average Phi Values for each face in order to determine its divergence from the numerical value of Phi. The distance values were calculated by taking the absolute value of the variance values, as statistical testing required the data to be in linear form, and reordered numerically.

Table 15: These are the faces' variances from Phi as compared to their respective average attractiveness ratings.

Variance vs. Attractiveness Rating

\begin{tabular}{|c|c|}
\hline Variance from Phi & Average Attractiveness Rating \\
\hline-0.137 & 5.178 \\
\hline-0.102 & 6.079 \\
\hline-0.081 & 6.257 \\
\hline-0.071 & 6.564 \\
\hline-0.039 & 7.307 \\
\hline-0.030 & 7.891 \\
\hline-0.017 & 8.366 \\
\hline-0.003 & 8.822 \\
\hline 0.010 & 8.634 \\
\hline 0.052 & 7.713 \\
\hline 0.114 & 5.772 \\
\hline 0.172 & 4.822 \\
\hline 0.185 & 4.376 \\
\hline 0.202 & 3.901 \\
\hline
\end{tabular}


Table 16: These are the faces' distances from Phi as compared to their respective average attractiveness ratings.

\begin{tabular}{|c|c|}
\hline \multicolumn{2}{|c|}{ Distance vs. Attractiveness Rating } \\
\hline Distance from Phi & Average Attractiveness Rating \\
\hline 0.202 & 3.901 \\
\hline 0.185 & 4.376 \\
\hline 0.172 & 4.822 \\
\hline 0.137 & 5.178 \\
\hline 0.114 & 5.772 \\
\hline 0.102 & 6.079 \\
\hline 0.081 & 6.257 \\
\hline 0.071 & 6.564 \\
\hline 0.039 & 7.307 \\
\hline 0.052 & 7.713 \\
\hline 0.030 & 7.891 \\
\hline 0.017 & 8.366 \\
\hline 0.010 & 8.634 \\
\hline 0.003 & 8.822 \\
\hline
\end{tabular}

The face with the least variance from Phi, Face 5, with a ratio of 1.615, received the highest average attractiveness rating of 8.822. In addition, the face with the greatest variance from Phi, Face 1, with a ratio of 1.820 , received the lowest average attractiveness rating of 3.901. There was no discernable discrepancy in the results between male and female faces.

\section{Data Analysis}

The Pearson Correlation Coefficient statistical test in Microsoft Excel was used to analyze the data and identify potential relationships or trends among the 14 data points. The formula for the test is $r=\left[n\left(\sum x y\right)-\left(\sum x\right)\left(\sum y\right)\right] /\left[\left[n \sum x^{2}-\right.\right.$ $\left.\left.\left.\left(\sum x\right)^{2}\right]\left[n \sum y^{2}-\left(\sum y\right)^{2}\right)\right]\right]^{1 / 2}$. Alpha values or critical values could not be identified because the Pearson Correlation Coefficient test does not generate these values. The observed $\mathrm{R}$ value for the data was calculated to be approximately 0.987 at an $\alpha=.01$ significance level, indicating a very strong correlation between distance from Phi and attractiveness rating. According to Figures 1 and 2, a relationship between closeness to Phi and average attractiveness rating was observed. The null hypothesis for this experiment stated that there is no relationship between expression of Phi in faces and perceived attractiveness, and the data rejects the null. The data indicated that as the distance from Phi decreased, the average attractiveness rating increased.

Table 18: Statistical Results. Shown here is the correlation-determining R value for the data.

\begin{tabular}{|l|l|l|l|}
\hline Test Statistic $(r)$ & $r^{2}$ & Correlation? & \\
\hline-0.987 & 0.974 & Yes & $\mathrm{H}_{\mathrm{O}}: \mathrm{r}=0$ \\
\hline
\end{tabular}




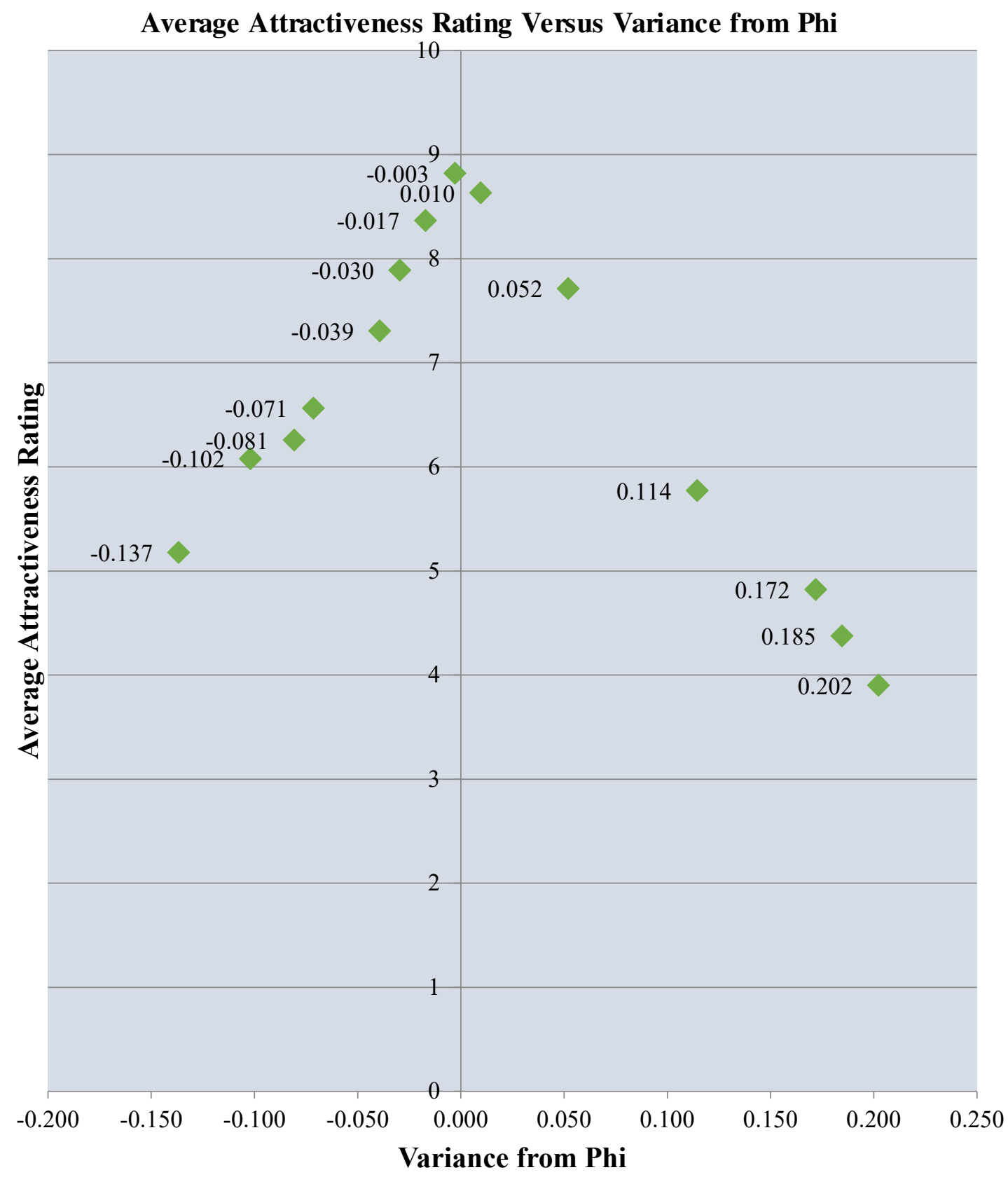

Figure 1: This graph compares the variances from Phi of the faces to their average attractiveness ratings. 


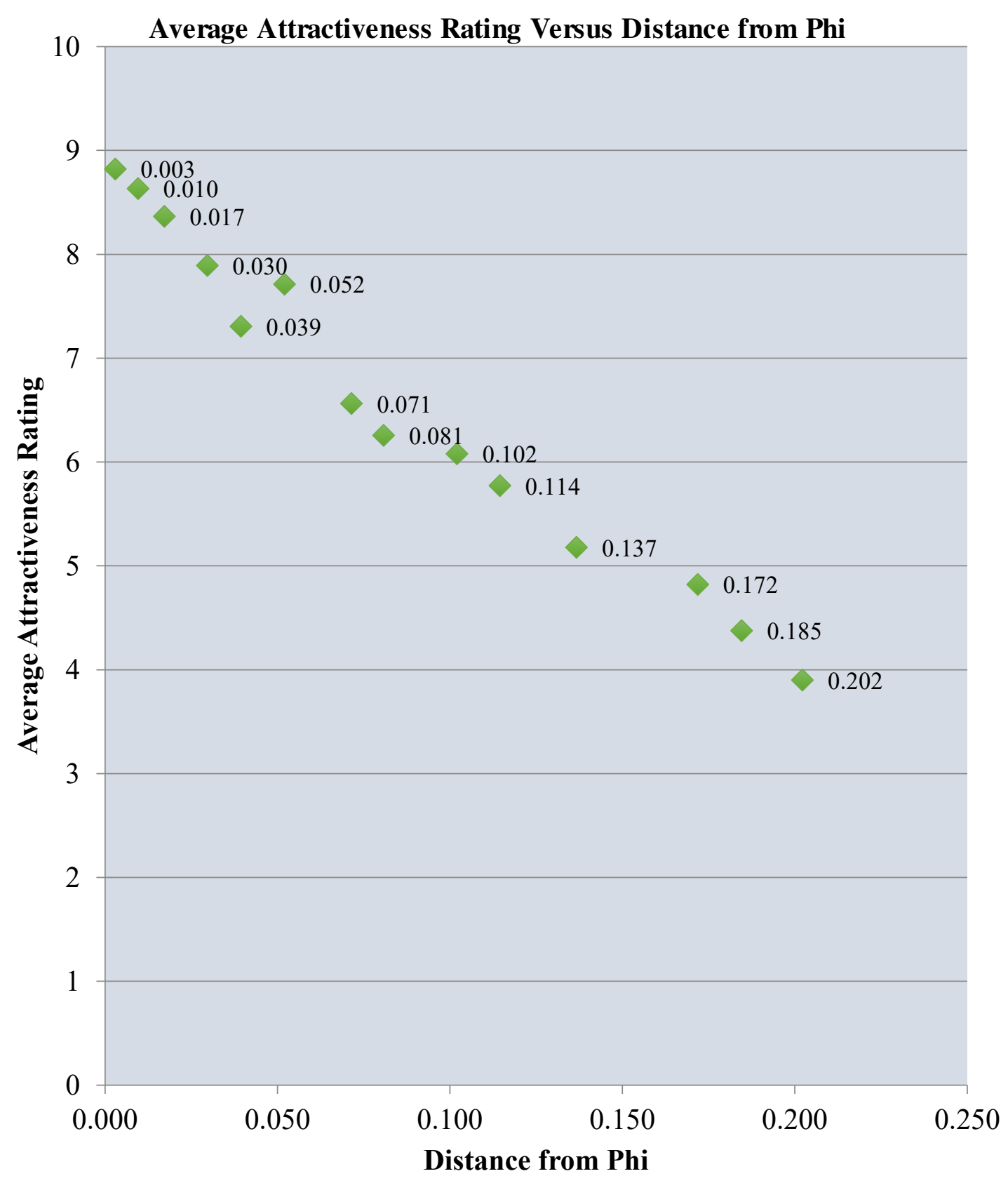

Figure 2: This graph compares the distances from Phi of the faces to their average attractiveness ratings.

\section{Summary}

The average Phi ratio for each face was calculated and then compared to its average attractiveness rating as established by the survey. The data show a strong correlation between the expression of Phi and perceived attractiveness. The face with the least variance from Phi, Face 5, received the highest average attractiveness rating. The face with the greatest variance from Phi, Face 1, received the lowest attractiveness rating. According to the data, as the distance of the average facial ratio away from Phi decreased, the average attractiveness rating increased. 


\section{Chapter Five}

\section{Conclusion}

\section{Summary of Findings}

The ratio of Phi $(\Phi)$, equal to approximately 1.618, is an observable phenomenon in nature and throughout history. Greek mathematician Euclid defined this relationship as a line in two parts, in which the ratio of the longer part to the shorter part is equal to the ratio of the total length over the longer part. The ancient Greeks thought of Phi as a mathematical representation of physical beauty. Although many studies have shown a correlation between Phi and perceived attractiveness, other studies have displayed no, or very weak, correlation. The purpose of this experiment was to investigate whether a significant relationship could be observed between Phi and perceived facial beauty. The question, "Does the expression of Phi play a significant role in the attractiveness of a face?" was investigated. It was hypothesized that if the features of a face closely fit the proportions of Phi, then survey respondents will give that face a higher attractiveness rating than a face whose features do not. The null hypothesis was that there is no correlation between Phi and perceived facial attractiveness.

Photographs of 14 faces were obtained from the Aberdeen 2D Face Set from Psychological Image Collection at Stirling, and landmark localization data for each of the 14 faces were collected. Individual and average facial ratios were calculated for each photograph, and a survey was conducted in which 100 participants were asked to rate the attractiveness of each face on a scale of 1-10. The average attractiveness rating of each face was then compared to the variance/distance of its average facial ratio away from the numerical value of Phi. A Pearson Correlation Coefficient statistical test was used to analyze the data to determine whether any significant trend could be identified.

\section{Conclusion and Discussion}

Based on the analysis of the data, faces with features whose proportions closely fit the ratio of Phi are perceived as more attractive than faces with features that do not. Faces with average facial ratios closer to Phi received higher average attractiveness ratings. The hypothesis stated that if the features of a face closely fit the proportions of Phi, then survey respondents will give that face a higher attractiveness rating than a face whose features do not. The results of the experiment supported the hypothesis.

The observed $\mathrm{R}$ value for the data was calculated to be approximately -0.987 at an $\alpha=.01$ significance level, indicating a very strong correlation between variance/distance away from Phi and average attractiveness rating. As distance away from Phi increased, average attractiveness rating decreased (as shown in Figures 1 and 2). The closer a face fit the proportions of Phi, the more attractive it was perceived to be. The null hypothesis that there is no correlation between Phi and perceived facial attractiveness was rejected. The results of the experiment indicated a relationship between Phi and perceived facial attractiveness, and support the existence of a mathematical standard of beauty in terms of Phi.

One weakness of the experiment was that landmark localization data for only 14 faces were measured and analyzed. Another weakness was that there were only 100 participants in the survey. These two factors could have contributed to reduced accuracy and reliability of the data. No unexpected findings or inconsistencies were observed or identified throughout the experiment or in the analysis of the data. 


\section{Recommendations}

In order to strengthen the reliability and precision of the data in this study, measures could be taken to enhance the experimental design. Expanding the data set by using more photographs and increasing the survey respondent pool would improve the accuracy of the experiment. In addition, utilizing a larger rating scale would increase the precision of the data by expanding the ratings-assignment parameters.

One variation of this experiment would be to test if the correlation between Phi and perceived facial attractiveness varies with the race of the individuals in the photographs, and/or with the races of the survey respondents. Another variation would be to test if survey respondents would rate faces differently if presented in a different order, or if presented with one gender at a time.

\section{Future Implications}

The relationship between Phi and perceived facial attractiveness could be applied to optimize facial reconstructive procedures and other cosmetic surgeries. If further significant correlation is identified, the ratio of Phi could be used as a guide to enhance ideal outcomes and patient satisfaction, as they relate to cosmetic procedures. The findings of this study support the existence of a mathematical standard of beauty in terms of Phi. These findings could be used as a basis for future investigation into correlation between attractiveness and other metrics. Based on the findings of this experiment, Phi could potentially be used as a quantitative measure to study the societal impacts of facial beauty and physical appearance.

\section{References}

Akan, S., Torgut, A., Oktay, H.. (2017). Effects of malocclusions on facial attractiveness and their correlations with the divine proportion. J Orofac Orthop. 78(5), p427-436.

Alam, M.K., Mohd Noor NF., Basri R., Yew T.F., Wen T.H.. (2015) Multiracial Facial Golden Ratio and Evaluation of Facial Appearance. PLoS ONE 10(11)

Ballester, J., Martínez Soriano, F., Ribera Vega, M., Font Ferrandis, J.. (2018). Assessment of facial harmony among Caucasian Spaniards 18 to 60 years of age and its relationship with the golden ratio. European Journal of Plastic Surgery. 41(2), p189-206.

Bashour, M. (2006). An Objective System for Measuring Facial Attractiveness. Plastic and Reconstructive Surgery. 118(3), p757-774.

Bușuat, V., Andrei, E. (2018). The Divine Proportion's Incidence in Facial and Dental Aesthetics. Romanian Journal for Multidimensional Education. 10(1) p155-70.

Cui, X., Cheng, Q., Lin, W., Lin, J., Mo, L.. (2019). Different influences of facial attractiveness on judgments of moral beauty and moral goodness. Scientific Reports. 9(1).

Eisenthal, Y., Dror, G., Ruppin, E.. (2006). Facial Attractiveness: Beauty and the Machine. Neural Computation. 18(1), p119-142. 
Fletcher, R. (2007). From graPhical to mathematical: The spiral of golden proportion. Australian Senior Mathematics Journal. 21(1), p46-52

Foos, P., Clark, M.. (2011). Adult Age and Gender Differences in Perceptions of Facial Attractiveness: Beauty is in the Eye of the Older Beholder. Journal of Genetic Psychology. 172(2), p162-175.

Ilić, I.; Stefanović, M.; Sadiković, D. (2018). Mathematical Determination in Nature - The Golden Ratio. Acta Medica Medianae 2018;57(3):124-129.

Nguyen, M., Saag, M., Le, V., Nguyen, T., Nguyen, B., Jagomägi, T.. (2016). The golden proportion in facial softtissues of Vietnamese females. Stomatologija. 8(3):80-85.

Özdemir, S., Çini, N. T.. (2019). Morphometric evaluation of the face: proximity to the golden ratio. International Journal of Experimental \& Clinical Anatomy. 13 (2), p191-192.

Pallett, P., Link, S., Lee, K.. (2010). New “Golden” Ratios for Facial Beauty. R Vision Research 50 (2), p149-153.

Sami Kaya, K., Türk, B., Cankaya, M., Seyhun, N., Uslu, B. (2018). Assessment of Facial Analysis Measurements by Golden Proportion. Brazilian Journal of Otorhinolaryngology Şişli Hamidiye Etfal Training and Research Hospital; 85, p494-501

Schmid, K., Marx, D., Samal, A.. (2008). Computation of a Face Attractiveness Index Based on Neoclassical Canons, Symmetry, and Golden Ratios. Pattern Recognition. 41(2008), p2710-2717

Veerala G, Sekhar Gandikota C, K Yadagiri, Manne R, Juvvadi S, Farah T, Vattipelli S, Gumbelli S. (2016) Marquardt's Facial Golden Decagon Mask and Its Fitness with South Indian Facial Traits. Journal of Clinical and Diagnostic Research. 10(4): p49-52 